extrinsic coagulation defects, intra-abdominal infection \& congenital abnormalities. These analyses were normal with diagnosis PVT of unknown cause; over $50 \%$ of cases have no known aetiology ${ }^{[2]}$. DH had complications including ascites, splenomegaly \& an oesophageal varix. He will need regular OGD follow up to check if change to varix or development of new varices, $79 \%$ of children with PVT have one upper gastrointestinal bleed in their lives ${ }^{[3]}$.

Conclusion Ascites is an unusual presentation with specific investigations. PVT is a rare cause of ascites with complications that require investigation.

[1] Karnasakul W, Thammasin I et al. Ascites in Children: A Single-Centre Experience of 27 years. Journal Paediatric Gastroenterology, Hepatology \& Nutrition, 64 (1),, 83-88. 2017

[2] Weiss B, Shteyer E, Vivante A, Berkowitz D, Reif S, Weizman Z, Bujanover Y, Shapiro R. Etiology and long-term outcome of extrahepatic portal vein obstruction in children. World J Gastroenterol. $2010 ; 16: 4968-72$.

[3] Ferri, Menezes P., et al. Portal vein thrombosis in children and adolescents: 20 years experience of a pediatric hepatology reference center. Arquivos de Gastroenterologia, 49(1), 69-76. 2012

\section{P334 CONGENITAL CYTOMEGALOVIRUS INFECTION}

\footnotetext{
${ }^{1,2}$ Alina Grama, ${ }^{2}$ Claudia Sirbe, ${ }^{2}$ Madalina Grigore, ${ }^{2}$ Adriana Bungardi, ${ }^{1,2}$ Tudor L Pop*. ${ }^{1}$ University of Medicine and Pharmacy 'Iuliu Hatieganu', Cluj-Napoca, Romania; ${ }^{2} 2 n d$ Paediatric Clinic, Cluj-Napoca, Romania
}

\subsection{6/archdischild-2019-epa.683}

Introduction Congenital cytomegalovirus (CMV) infection is the most common congenital infection, with an incidence of $0.5 \%$ among neonates. Mother to child CMV transmission could be transplacental (mostly), at delivery or after birth, through exposure to breast milk. The clinical picture may range from asymptomatic disease to severe form of illness, sometimes occurred immediately after birth. These symptomatic infants have a high risk for neurologic sequelae, including sensorineural hearing loss, mental retardation, microcephaly, development delay, seizure disorders, and cerebral palsy. Congenital CMV is the most common cause of acquired hearing loss in childhood.

Clinical cases We report the clinical and laboratory parameters of three patients with severe congenital CMV infection. In all the maternal infection was asymptomatic and TORCH serology before/during pregnancy was not performed.

The first patient was a preterm boy (born at 28 weeks of gestation with the weight of $1,190 \mathrm{~g}$ ) with cholestatic hepatitis and complex perinatal pathology, necrotizing enterocolitis, recurrent episodes of apnoea and bradycardia, severe anaemia and thrombocytopenia in the context of CMV infection. The second patient was a girl, born at 37 weeks of gestation with intrauterine growth retardation (weight 2,420 g), who developed immediately after birth CMV pneumoniae, cholestatic hepatitis and severe cytopenia. Also, the audiogram was suggestive for hearing impairment. The third patient was a preterm girl, born at 35 weeks of gestation, weight 2,600 g, with cerebral vasculopathy and splenomegaly after intrauterine CMV infection.

In all these patients, the infection was confirmed by positive serological IgM-CMV antibodies and by quantitative CMV
PCR assay in plasma and all of them received antiviral therapy with ganciclovir.

Conclusions Congenital CMV is an important cause of morbidity in children. Early detection of CMV infection by CMV PCR or culture from saliva or urine and a prompt treatment can prevent life threatening complications like acute liver failure, pneumonitis or sepsis-like syndrome or can improve hearing and neurological development. All children with congenital CMV should be periodically monitored to prevent hearing loss or other neurocognitive sequelae.

\section{P335 'LIPID TRIAD ‘ IN PATIENTS WITH CHRONIC PANCREATITIS IN COMBINATION WITH ARTERIAL HYPERTENSION}

Olesya Horlenko*, Emilia Arkhiy, Lyubomyra Prylypko, Oxana Moscal, Maria Derbak , Bogdan Halay. Uzhhorod National Universiry, Uzhhorod, Ukraine

\subsection{6/archdischild-2019-epa.684}

Introduction Modern scientific research in the field of medical knowledge has repeatedly confirmed that the issue of 'comorbidity' is becoming widespread. More and more rare occurrences of the mononozological course of various diseases and the further, the more often - variants of a comorbid or multi-morbid.

Materials In order to achieve this goal, we carried out a general clinical, laboratory and instrumental examination of 102 patients with comorbid pathology of CP and GC that were in the hospital treatment of Khust district hospital during 20172018. The average age of the examined patient was $51 \pm 10$ years.

Results Concerning the features of lipid metabolism, the results obtained by us confirm the presence of 'lipid triad' in patients with Chronic Pancreatitis (CP) in combination with Arterial Hypertension a generalized persistence of inflammatory changes at the level of vascular endothelium. It is difficult to say with certainty that the primary focus in the examined group of patients, or dislipoproteinemia against the background of an existing $\mathrm{CP}$ with $\mathrm{AH}$, but it is possible to clearly state the imbalance in the system of lipid homeostasis, which ultimately changes the activity of all organs and systems. The results of the study, which reproduce the changes in Apolipoprotein levels, were as follows: in the main group, the concentration of Apo A1 decreased to $0.85 \pm 0.11 \mathrm{~g} / \mathrm{l}$ against $1.08 \pm 0.12 \mathrm{~g} / \mathrm{l}$ in the control group $(\mathrm{p}<0,05)$, and the excessive level of proatherogenic $A p o B$ in the group of patients with $\mathrm{CP}$ and $\mathrm{AH}-1.46 \pm 0.23 \mathrm{~g} / \mathrm{l}$, and in practically healthy individuals $-0.99 \pm 0.24 \mathrm{~g} / \mathrm{l}(\mathrm{p}<0,05)$

In addition to the absolute values of lipidogram rates, in order to assess the presence or potential risk of developing atherosclerotic vascular damage, the ratio of Apo B/Apo A1 and the atherogenicity index that were higher in the group of patients with CP and GC: Apo B/Apo A1 - 1, $77 \pm 0.46$ versus $0.92 \pm 0.14$ (in the control group $(\mathrm{p}<0,05)$ and IA - in the I group $-5,42 \pm 2,25$ versus $2,4 \pm 1,02(\mathrm{p}<0,05)$. The latter indicators are necessary to determine the further treatment of patients and control of the prescribed therapy effectiveness.

Conclusion The results of the study carried out during the hospitalization period in the main group indicate an increase in concentrations of proatherogenic fractions of lipids and apolipoproteins (LDL, LPDH, Apo B) and reduction of antiatherogenic (HDL, Apo A1). The atherogenicity index and the 
ratio of Apo B/Apo A1 was also the highest than the norm. Therefore, we can assume that dyslipidemic disorders, without of effective means of correction, will contribute to the progression of both - $\mathrm{CP}$ and $\mathrm{AH}$.

\section{P336 HYPOALBUMINEMIA AND OEDEMA IN A 4 MONTH-OLD BOY}

${ }^{1}$ Deirdre O'Sullivan*, ${ }^{1}$ Rincy Koshy, Declan Cody 1,2, 1,2Billy Bourke. 'Our Lady's Children Hospital, Crumlin, Dublin, Ireland; ${ }^{2}$ University College Dublin, Dublin, Ireland

\subsection{6/archdischild-2019-epa.685}

Aims Primary intestinal lymphangiectasia (PIL) is a well-recognized congenital abnormality of the lymphatic system leading to protein-losing enteropathy (PLE). It is an uncommon disorder seldom seen in clinical practice. The prevalence is unknown. The objective of this case report is to describe the clinical presentation and diagnosis of PIL.

Methods A detailed chart review was performed. Data extracted from the medical records included presenting complaint, disease progression, laboratory results, imaging and clinical measurements.

Results The patient was a 4 month old boy, who presented to the emergency department in Our Lady's Children Hospital, Crumlin (OLCHC) with a 3 week history of bilateral lower limb swelling, easy bruising and a change in stool consistency. The baby was passing watery secretions prior to stool. Stool consistency was clay- like with mucous. Initial bloods showed a severe coagulopathy (PT 204.6 secs, APTT 80.4 secs), severe electrolyte disturbances $(\mathrm{Na}+128 \mathrm{mmol} / \mathrm{l}, \mathrm{K}+2.6 \mathrm{mmol} / \mathrm{l})$, mild transaminase elevation (AST $87 \mathrm{U} / 1$, ALT $49 \mathrm{U} / \mathrm{l}$ ), metabolic acidosis ( $\mathrm{pH} 7.293$, pCO2 4.75, HCO3- 16.9) and profound hypoalbuminemia $(13 \mathrm{~g} / \mathrm{l})$. The patient was admitted for electrolyte replacement and for further investigation of the underlying cause of the hypoalbuminemia and oedema. Stool alpha- 1-antitrypsin $(5.66 \mathrm{mg} / \mathrm{g})$ was markedly elevated confirming protein-losing enteropathy. Further investigations including endoscopic biopsies and allergy testing excluded other enteropathies such as coeliac disease, inflammatory bowel disease, congenital enteropathies and allergic enteritis. As in this case PIL pathology is often mid small bowel and beyond the reach of endoscopic biopsies.

Conclusion Hypoalbuminemia can manifestation from a variety of clinical disorders. This patient had an extensive work-up to exclude other possible causes. Once PLE was confirmed, further investigations were required to identify the underlying cause. The main laboratory findings that supported the diagnosis of PIL included hypoproteinaemia, protein losing enteropathy, lymphopenia, panhypogammaglobulinemia, malabsorption of fat-soluble vitamins and, ultimately, response to a low long chain triglyceride containing diet.

\section{P337 CURRENT PRACTICES FOR THE TREATMENT OF GASTROENTERITIS IN CHILDREN IN WEXFORD GENERAL HOSPITAL}

1,2Muhammad Zia*, 'Mohammad Taha, 'Maybelle wallis. 'Wexford general Hospital, wexford, Ireland; ${ }^{2}$ University Maternity Hospital Limerick, limerick, Ireland

10.1136/archdischild-2019-epa.686

Background Gastroenteritis(GE) is common in children.
Aim of the audit 1.To identify if rehydration treatment given was appropriate for the degree of dehydration?

2.To look how many children with gastroenteritis received saline boluses in emergency department or in ward when it was not indicated clinically.

3.To find out if we are using modes of rehydration other an intravenous in children with mild to moderate dehydration.

4.Whether ORS solution was used by parents before coming to hospital?

Methodology All children $<16$ years who presented to emergency department or were admitted in Gabriel ward with symptoms of diarrhoea or vomiting were included in the audit. The audit period was from $06 / 02 / 2018$ to 31/03/2018.

Results 1.Total 24 patients were collected from ED 20(83\%) and Gabriels ward4(17\%).

2. Out of them $11(45.8 \%)$ were female and 13(54.2\%)were male patients

3. Age ranges from 5 weeks to 11 years.13(54\%)patients were below 2 years of age.

4. All 24(100\%) patients had vomiting and $17(70.3 \%)$ patients had diarrhoea and vomiting at presentation.8(33.3\%) patients had temperature also.

5. $4(16.6 \%)$ patients had been given ORS before coming to hospital.

6. $18(75 \%)$ patients had no dehydration and 6(25\%) with clinical dehydration. No patients presented in shock.

7. Regarding mode of rehydration $20(83.3 \%)$ patients were given trial of oral rehydration and $4(16.7 \%)$ were given IV rehydration after failed oral challenge

8. IV bolus of Normal saline were given to $2(8.3 \%)$ patients.

9. Ondenstron was given to $18(75 \%)$ patients. $4(16.7 \%)$ patients failed oral challenge.

10. Nasogastric rehydration was not given to any of the patients.

Conclusions The use of ORS before coming to hospital was negligible. If used appropriately at home can reduce the hospital attendances. The NG mode of rehydration was not tried in any of our patients. By adapting NG mode of Rehydration we can avoid IV rehydration. The saline boluses were not indicated in patients who received them as they were not in severe dehydration or shock.

Recommendations and action plan 1. Education of GPS and parents about the importance of use of ORS once started with the symptoms of GE to avoid hospital admissions.

2. Awareness among NCHDs and paediatric nurses to use alternate routes of rehydration other than IV.

3. Patients presenting to ED department with no signs of dehydration should be encouraged for use of ORS at home.

4. Current practice of giving saline bolus in pateints who are not shocked should be discouraged.

\section{P338 COIN VS BATTERY - IMAGING OF FOREIGN BODIES}

Megan Sheppard*. Lady Cilento Children's Hospital , Brisbane, Australia

\subsection{6/archdischild-2019-epa.687}

A previously well 2 year old female presented to a peripheral emergency department following the ingestion of a foreign body. Her parents reported witnessing her swallow a coin, 2 days prior to presentation. She subsequently developed 24 hours of non-bilious vomiting followed by epigastric pain and 